\title{
O impacto da Psicomotricidade no tratamento de crianças com transtorno do Espectro Autista: revisão integrativa
}

\author{
The impact of Psychomotricity in the treatment of children with Autism Spectrum an: \\ integrative review
El impacto de la Psicomotricidad en el tratamiento de niños con trastorno del Espectro Autista: revisión integrativa

Érica Monteiro Oliveira ${ }^{1 *}$, Francisca Tatiana Dourado Gonçalves ${ }^{2}$, Marcio Marinho Magalhães ${ }^{3}$, Helena Morgana Sousa Do Nascimento ${ }^{4}$, Izabel Cristina Vale de Carvalho ${ }^{5}$, Ana Valéria Lopes Lemos $^{6}$, Érika Castelo Branco Said ${ }^{7}$, Maria de Jesus Martins de Andrade Silva Cunha ${ }^{8}$, Zaira Arthemisa Mesquita Araujo ${ }^{8}$, Pedro Wilson Ramos da Conceição ${ }^{8}$, Emanuel Monteiro Oliveira ${ }^{9}$, Lucas Gabriel Ribeiro Limeira ${ }^{9}$, Carlos Alberto Sousa Silveira ${ }^{10}$, Murilo Simões Carneiro ${ }^{11}$.

\section{RESUMO}

Objetivo: realizar uma revisão integrativa para identificar as contribuições da Psicomotricidade como uma técnica de fortalecimento do desenvolvimento psicomotor em crianças com autismo. Métodos: Trata-se de uma revisão integrativa de caráter bibliográfica exploratória e descritiva dos resultados apresentados. Foi realizada uma busca referente ao tema abordado em artigos publicados entre 2010 a 2018 em português e inglês. As bases de dados utilizadas foram "Scientific Electronic Library Online (SCIELO)", "Medical Literature Analysis and Retrieval System Online (MEDLINE)" e "Literatura Latino-Americana e do Caribe em Ciências da Saúde (LILACS)" Foram utilizados 9 artigos para este trabalho. Resultados: Estudos mostram que a Fisioterapia através dos princípios da contribuição da Psicomotricidade tem benefícios que permite que a criança constitui-se na principal via de expressão do seu mundo interno e externo, onde obteve a melhora da qualidade de vida das crianças com Transtorno do Espectro do Autismo (TEA), proporcionou benefícios positivos nos sistemas, motor, cognitivo, sensorial, no desenvolvimento do ritmo, esquema corporal, postura, equilíbrio, coordenação motora, estruturação espacial, orientação temporal e interação social. Conclusão: Através desta revisão foi possível perceber que a fisioterapia tem um papel satisfatório onde se pode observar a importância da utilização da psicomotricidade no desenvolvimento, pois contribuem para evolução e estabilidade no equilíbrio, coordenação motora, hábitos de vida e interação social das crianças com diagnóstico de transtorno do espectro autista.

Palavras-chave: Fisioterapia, Psicomotor, Crianças, Autismo.

\footnotetext{
ABSTRACT

Objective: To conduct an integrative review to identify the contributions of Psychomotricity as a technique for strengthening psychomotor development in children with autism. Methods: This is an integrative review of an exploratory and descriptive bibliographical character of the results presented. A search was made regarding the topic addressed in articles published between 2010 and 2018 in Portuguese and English. The

1 Centro Universitário de Ciências e Tecnologias do Maranhão (UNIFACEMA), Caxias (MA). ${ }^{*} E$-mail: fisioericamonteiro19@gmail.com

${ }^{2}$ Faculdade de Ensino Superior do Piauí (FAESPI), Teresina (PI).

${ }^{3}$ Faculdade Einstein (FACEI), Caxias (MA).

${ }^{4}$ Centro Universitário de Ciências e Tecnologias do Maranhão (UNIFACEMA), Caxias (MA).

5 Universidade de Brasília (UNB), Brasília (DF).

6 Universidade Estadual do Piauí (UESPI), Teresina (PI).

${ }^{7}$ Faculdade Inspirar (FACULDADE INSPIRAR), Teresina (PI).

${ }^{8}$ Universidade Federal do Piauí (UFPI), Teresina (PI).

${ }^{9}$ Centro Universitário de Ciências e Tecnologias do Maranhão (UNIFACEMA), Caxias (MA).

${ }^{10}$ Associação de Ensino Superior do Piauí (AESPI), Teresina (PI).

11 Faculdade Unyleya (UNYLEYA), Rio de Janeiro (RJ).
} 
databases used were" Scientific Electronic Library Online (SCIELO)", "Medical Literature Analysis and Retrieval System Online (MEDLINE)" and "Literatura Latino-Americana e do Caribe em Ciências da Saúde (LILACS)". 9 articles were used for this job. Results: Studies show that physical therapy through principles of the contribution of Psychomotricity has benefits that allow the child is the main way of expressing his internal and external world, improvement in the quality of life of children with Autism Spectrum (TEA), provided positive benefits in systems, motor, cognitive, sensory, in the development of the rhythm, corporal scheme, posture, balance, motor coordination, spatial structuring, temporal orientation and social interaction. Conclusion: Through this review it was possible to that physiotherapy has a satisfactory role in which it is possible to observe the importance of use of psychomotricity in development, since they contribute to the evolution and stability in balance, motor coordination, life habits and social interaction of children diagnosed with autism spectrum disorder.

Key words: Physiotherapy, Psychomotor, Children, Austim.

\section{RESUMEN}

Objetivo: realizar una revisión integradora para identificar las contribuciones de la psicomotricidad como técnica para fortalecer el desarrollo psicomotor en niños con autismo. Métodos: Se trata de una revisión integrativa de carácter bibliográfica exploratoria y descriptiva de los resultados presentados. Se realizó una búsqueda referente al tema abordado en los artículos publicados entre 2010-2018 en portugués y en Inglés. Las bases de datos se utilizaron "Scientific Electronic Library Online (SCIELO)", "Medical Literature Analysis and Retrieval System Online (MEDLINE)" y "Literatura Latino-Americana e do Caribe em Ciências da Saúde (LILACS)". Se utilizaron 9 artículos para este trabajo. Resultados: Estudios muestran que la Fisioterapia a través de los principios de la contribución de la Psicomotricidad tiene beneficios que permite que la el niño se constituye en la principal vía de expresión desu mundo interno y externo, donde obtuvo la mejora de la calidad de vida de los niños con trastorno del sueño Espectro del Autismo (TEA), proporcionó beneficios positivos en los sistemas, motor, cognitivo, sensorial, en el desarrollo del ritmo, el esquema corporal, la postura, equilibrio, coordinación motora, estructuración espacial, orientación temporal y interacción social. Conclusión: Através de esta revisión fue posible percibir que la fisioterapia tiene un papel satisfactorio donde se puede observar la importancia de la utilización de la psicomotricidad en el desarrollo, pues contribuyen a la evolución y estabilidad en el equilibrio, coordinación motora, hábitos de vida e interacción social de los niños con diagnóstico de trastorno del espectro autista.

Palabras clave: Fisioterapia, Psicomotor, Ninõs, Autismo.

\section{INTRODUÇÃO}

O Transtorno do Espectro Autista (TEA) é definido por meio de comprometimentos precoces no desenvolvimento sócio comunicativo, assim como pela presença de comportamentos repetitivos e estereotipados, segundo (AMERICAN PSYCHIATRIC ASSOCIATION, 2013).

Segundo o autor Dias S (2015), diz que nossa sociedade ainda busca conhecimentos e tem muito para evoluir quando falamos sobre o Autismo ou como caracterizamos atualmente nos Transtornos do Espectro Autista (TEA). De acordo com Santos THF e Fernandes FDM (2012), o autismo pode ser percebido nos primeiros anos de vida, por meio de algumas características típicas, sendo de extrema importância o diagnóstico precoce, para um tratamento e estimulação mais eficaz nos aspectos psicológicos, biológicos e sociais, trazendo uma maior qualidade de vida para a criança autista. Uma das características que é possível identificarem é no contato visual, pois o bebê não consegue manter o contato visual por muito tempo com uma pessoa.

No estudo de Backes BZ et al. (2013) O Transtorno do Espectro do Autista é uma condição neurodesenvolvimento que se apresenta no início dos primeiros anos de vida da criança. Dados epidemiológicos demonstram uma prevalência de um em cada 150 nascimentos, sendo que esta vem aumentando nas últimas décadas. Vem acontecendo um aumento fora do comum de crianças diagnosticadas com o Transtorno do Espectro do Autismo (TEA).

Segundo Locatelli PB e Santos MFR (2016) devido os sintomas estarem presentes antes dos 3 anos de idade, dependendo da gravidade do comprometimento, torna possível fazer o diagnóstico por volta dos 18 
meses de idade. O diagnóstico precoce do autismo torna-se importante para que haja um direcionamento do mesmo ao tratamento mais adequado as suas necessidades, fazendo toda a diferença, com técnicas e terapias para estimular a criança. Reconhecendo a natureza dimensional desse conjunto de condições que fazem parte do espectro e as controvérsias em relação ao diagnóstico diferencial entre elas, a quinta edição do Manual Diagnóstico e Estatístico de Transtornos Mentais (DSM-5). Onde o universo da criança autista é uma realidade complexa que engloba vários conceitos distintos, mas que sempre se cruzam em determinados pontos (AMERICAN PSYCHIATRIC ASSOCIATION - APA, 2014).

O TEA é uma condição que tem início precoce e cujas dificuldades tendem a comprometer o desenvolvimento do indivíduo, ao longo de sua vida, ocorrendo uma grande variabilidade na intensidade $\mathrm{e}$ forma de expressão da sintomatologia, nas áreas que definem o seu diagnóstico. Atualmente, o TEA é compreendido como uma síndrome comportamental complexa que possui etiologias múltiplas. Por ser um distúrbio com diferentes níveis de comprometimento o desenvolvimento da criança tende a ser menos comunicativo (RUTTER ML 2011).

Os autores Azevedo A e Gusmão M (2016) relatam que crianças com diagnóstico tardio de transtorno autista apresentaram problemas no padrão motor. A fisioterapia trabalhando com a psicomotricidade traz a melhora no padrão motor desenvolvendo melhora na marcha e no equilíbrio.

De acordo com Zimmer PM et al, (2018), o termo Psicomotricidade tem como significado "A capacidade de determinar e coordenar mentalmente os movimentos corporais; a atividade ou conjunto de funções motoras. A atividade da criança é elementar suas primeiras manifestações de comportamento são essenciais dando início na ordem motora e por fim passam a ser a ordem mental.

A Psicomotricidade é uma possibilidade de intervenção com crianças autistas, que fortalecem a interiorização da criança ao se movimentar em torno de si mesma e dificultam a relação desta com o mundo com psicomotricidade traz a melhora no padrão motor desenvolvendo melhora na marcha e no equilíbrio (GONÇALVES IAM 2012). De acordo com Andrade FFD (2014), a prática da terapia psicomotora abrange aspectos que relacione o indivíduo aos sentimentos, traumas e sua ligação à expressão através do corpo, 0 indivíduo relaxa e trabalhe o sentimento de forma que realize um trabalho de controle de sentimento auxiliando na socialização. A psicomotricidade é um fator de grande relevância para o desenvolvimento da criança, pois, a partir dela, tem-se a capacidade de desenvolver as habilidades dos pacientes no espaço que eles ocupam e na própria vida.

No estudo de dos Anjos CC, et al. (2017) relata que, uma das formas de se trabalhar o desenvolvimento global do indivíduo e os possíveis desvios por meio da intervenção psicomotora, área de atuação do profissional de Fisioterapia. Assim, a Fisioterapia pode atuar na criança com Transtorno do Espectro Autista na ativação dos níveis sensorial e motor, buscando melhorar a concentração, a memória e as habilidades motoras, como a coordenação e o equilíbrio. O papel da Fisioterapia é importante, pois ajuda no desenvolvimento motor da criança, trazendo a melhora da qualidade de vida.

De acordo com Kumamoto L (2012); À medida que estas trocas, mesmo que elementares, permitem estabelecer um vínculo de confiança, as interações tornam-se mais claras e começam a surgir iniciativas onde é possível identificar uma intencionalidade, quando, por exemplo, a criança chuta uma bola ou simplesmente olha em direção ao terapeuta, à professora ou a outra criança em sala de aula ou qualquer outra situação do grupo. Dependendo da situação, o profissional introduz gradativamente nos comentários, referências afetivas sobre sentimentos de alegria ou desagrado que a criança, porventura, manifesta em alguma situação.

Esta pesquisa teve como objetivo principal verificar as contribuições da utilização da Psicomotricidade como uma técnica de fortalecimento do desenvolvimento psicomotor em crianças com autismo.

\section{METODOLOGIA}

Este estudo utiliza como método a revisão integrativa, a qual tem como finalidade reunir e resumir o conhecimento científico. Para permitir buscar, avaliar e sintetizar as evidências disponíveis para contribuir 
com o desenvolvimento do conhecimento na temática. Foram incluídos trabalhos disponíveis nas seguintes bases de dados: "Scientific Electronic Library Online (SCIELO)", "Medical Literature Analysis and Retrieval System Online (MEDLINE)" e "Literatura Latino-Americana e do Caribe em Ciências da Saúde (LILACS)" E como critérios de inclusão: Estudos publicados no período de 2010 a 2018, nos idiomas português e inglês. Foram excluídos resumos, artigos com equívocos metodológicos, teses, dissertações, artigos de revisão e que não contivessem ano de publicação, volume e número em revista.

Os descritores utilizados foram: "Fisioterapia", "Psicomotor"; "Crianças"; "Autismo". Após as buscas nas bases de dados utilizando a associação (Equoterapia AND Paralisia Cerebral AND Fisioterapia), foram encontrados 1500 artigos, mediante aplicação dos critérios de inclusão ficaram 500 estudos publicados. Destes, após a aplicação critérios de exclusão, restaram 58 publicações. Dessas, 9 publicações apresentavam metodologia, temática e objetivos condizentes com os objetivos da pesquisa atual (Figura 1).

Figura 1 - Representação gráfica da aplicação dos critérios de inclusão e exclusão.

"Fisioterapia AND Psicomotor", "Psicomotricidade AND Autismo"

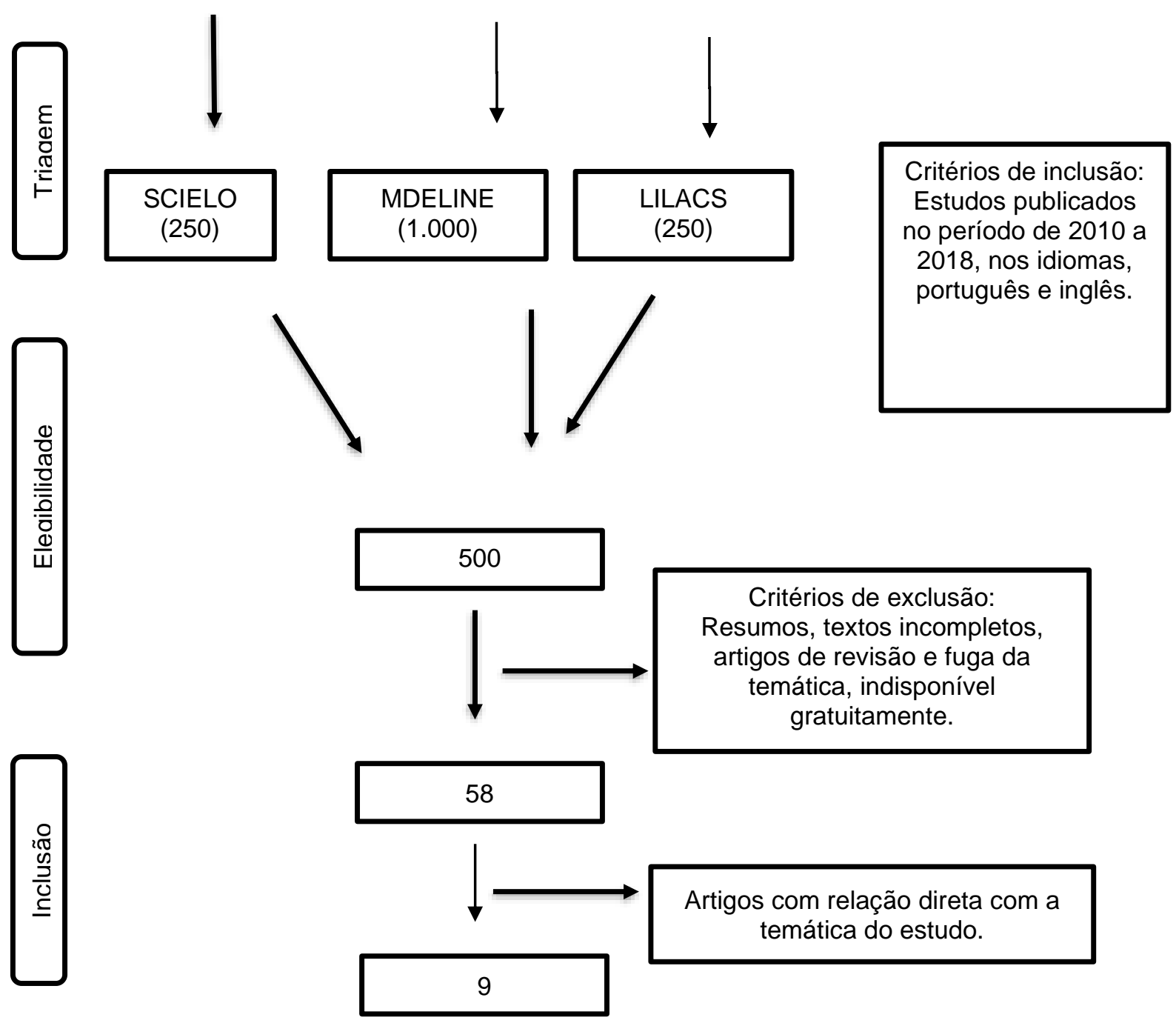

Fonte: Oliveira ME, Gonçalves DTF, Magalhães MM, et al., 2019. 


\section{RESULTADOS E DISCUSSÃO}

Os resultados a seguir mostram na Tabela 10 ano de publicação dos artigos encontrados, na Tabela 2 o idioma e no Quadro 1 mostra o ano, autor e resultado encontrado em cada artigo.

Tabela 1 - Distribuição dos estudos incluídos, segundo o ano de publicação.

\begin{tabular}{ccc}
\hline Ano da publicação & Número absoluto & $\%$ \\
\hline 2018 & 4 & 40,0 \\
2017 & 3 & 30,0 \\
2016 & 1 & 15,0 \\
2010 & 1 & 15,0 \\
TOTAL & 9 & $100 \%$ \\
\hline
\end{tabular}

Fonte: Oliveira ME, Gonçalves DTF, Magalhães MM, et al., 2019.

Tabela 2 - Distribuição dos artigos de acordo com o idioma.

\begin{tabular}{lcc}
\hline Idioma & Número absoluto & $\%$ \\
\hline Português & 8 & 85,0 \\
Inglês & 1 & 15,0 \\
\hline TOTAL & 9 & $100 \%$ \\
\hline
\end{tabular}

Fonte: Oliveira ME, Gonçalves DTF, Magalhães MM, et al., 2019.

No Quadro 1 apresenta a distribuição das publicações quanto ao ano, autores, e principais resultados relacionados as Contribuições da Psicomotricidade em crianças com Transtorno do Espectro Autista.

Quadro 1 - Publicações relacionadas às contribuições da Psicomotricidade em crianças com TEA.

\begin{tabular}{|c|c|c|c|}
\hline Trabalhos & Ano & Autor(es) & Resultados \\
\hline T1 & 2018 & Dos Santos FCÉ e Melo RT & $\begin{array}{l}\text { Podem-se perceber os efeitos positivos dessa } \\
\text { intervenção no desenvolvimento da criança } \\
\text { melhorando o equilíbrio e coordenação } \\
\text { e a melhora da qualidade de vida }\end{array}$ \\
\hline T2 & 2018 & Souza PT e Cruz PLC & $\begin{array}{l}\text { O trabalho com a psicomotricidade auxiliou na } \\
\text { realização das atividades da vida diária } \\
\text { (AVD's) promovendo a autonomia ao } \\
\text { Individuo. }\end{array}$ \\
\hline T3 & 2018 & Dos Anjos CC, et al. & $\begin{array}{l}\text { Promoveu benefícios na função motora } \\
\text { estimulando o desenvolvimento equilíbrio e } \\
\text { coordenação da criança. } \\
\text { A psicomotricidade teve resultados positivos }\end{array}$ \\
\hline T4 & 2018 & Cordeiro LC e da Silva D & $\begin{array}{l}\text { movimentos do corpo na relação } \\
\text { afetiva, podendo ainda se expressar } \\
\text { e ser compreendida. }\end{array}$ \\
\hline T5 & 2017 & Dos Anjos CC, et al. & $\begin{array}{l}\text { Resultou-se que a Fisioterapia através dos } \\
\text { princípios da psicomotricidade teve } \\
\text { fundamento no padrão motor e sensitivo } \\
\text { melhorando a qualidade de vida. }\end{array}$ \\
\hline T6 & 2017 & Souza DC, et al. & $\begin{array}{l}\text { Teve melhora na resistência e padrão motor } \\
\text { da criança. }\end{array}$ \\
\hline $\mathrm{T7}$ & 2017 & Cruz BDQ e Pottker CA & $\begin{array}{l}\text { No desenvolvimento psicomotor com a } \\
\text { Equoterapia para criança autista ajudou } \\
\text { na postura, equilíbrio coordenação motora, } \\
\text { orientação temporal e estruturação } \\
\text { espacial. }\end{array}$ \\
\hline T8 & 2016 & Trindade NG e Farias NC & $\begin{array}{l}\text { Proporcionou melhora no aprendizado } \\
\text { cognitivo, motor e interação social. }\end{array}$ \\
\hline T9 & 2010 & Falkenbach AP, et al. & $\begin{array}{l}\text { Teve efeitos positivos nos aspectos motores, } \\
\text { cognitivos e sensitivos. }\end{array}$ \\
\hline
\end{tabular}

Fonte: Oliveira ME, Gonçalves DTF, Magalhães MM, et al., 2019. 
Para dos Santos FCÉ e Melo RT (2018), relatam em seu estudo, que a intervenção através da psicomotricidade, a criança obteve os dois resultados positivos ao lançar uma bola, e ao pentear o cabelo, lado direito e esquerdo, $O$ conhecimento de tais parâmetros psicomotores alterados forneceu bons resultados para o conhecimento do caso e melhor acompanhamento evolutivo. A educação através da psicomotricidade é de extrema importância para o progresso da criança ao longo de seu crescimento no ato de educar na mente e o corpo simultaneamente no momento da formação da personalidade.

Certificando com o estudo de Souza PT e Cruz PLC (2018), onde afirmam que foi realizado o seu estudo com um menino, através das atividades psicomotoras foi uma vivência muito produtiva e de descobertas, avanço e retrocesso e de aprendizagem e que a escolha das atividades cognitivas e motoras, inseridas pelo núcleo familiar no tratamento do TEA, deve levar em consideração as especificidades de cada caso. Isso auxilia para que o pequeno aumente seus recursos frente aos desafios surgidos na vida escolar, no dia a dia do ambiente doméstico em outras etapas de sua autonomia.

Dos Anjos CC et al. (2018), relatam que através dos resultados positivos, ele afirma que os Fisioterapeutas podem trabalhar as crianças com TEA focando em habilidades motoras básicas, como: rolar, sentar-se, ficar de pé, andar. Essas atividades não são apenas importantes para o desenvolvimento físico, mas também para o engajamento social. Portanto o trabalho da Fisioterapia através da psicomotricidade trás vários benefícios para a criança com o autismo.

Cordeiro LC e da Silva D (2018), enfatizam em seu estudo, que pode-se perceber que a Psicomotricidade vem estimulando e melhorando a coordenação motora de acordo com o objetivo desejado da criança, como, coordenação motora fina e grossa, o trabalho da psicomotricidade também motiva as crianças na descoberta de suas expressões, além de impulsionar a ação criativa e da emoção e com isso trás benefícios na comunicação para a interação social.

Diante do estudo de dos Anjos CC et al. (2017), descrevem em sua pesquisa que os resultados do comprometimento motor nas habilidades motoras básicas em crianças com Transtorno do Espectro Autista têm sido observados já na primeira infância e nos primeiros 2 anos de vida, que significa que todas as crianças autistas têm sintomas diferentes e é neste sentido que se vincula o nosso interesse em abordar esta temática. $O$ trabalho da fisioterapia através da psicomotricidade traz benefícios importantes para a criança, onde ela desenvolve o seu padrão motor e cognitivo.

Corroborando com a afirmativa Souza DC et al, (2017), afirmam que obteve um ótimo resultado relatando que os benefícios das atividades desenvolvidas como psicomotricidade e da natação são notados já nas primeiras semanas de participação no projeto, pois, as atividades no meio aquático, estimulam o apetite, acalma e melhora a resistência das crianças. E com isso melhorar a qualidade de vida das pessoas com autismo, envolvidas nas atividades de Psicomotricidade aquática e a socialização partindo do princípio das possibilidades individuais.

Conforme Cruz BDQ e Pottker CA (2017), a Equoterapia através da Psicomotricidade traz benefícios para a criança autista, como: desenvolvimento de esquema corporal, devido a interação do corpo com o meio, ajudando na postura e equilíbrio; coordenação motora, utilizando os músculos maiores ou menores para controlar os movimentos do corpo. Onde com isso trás resultados positivos e avanços nas relações sócio educativas no aprendizado e desenvolvimento psicomotor das crianças com TEA.

No artigo de Trindade NG e Farias NC (2016), afirmam o relato em seu estudo que tendo a música como auxílio na fisioterapia relacionando com a psicomotricidade em indivíduos com autismo, notou-se que houve uma evolução no aspecto qualitativo, visando ganhos no comportamento, denotando traços mais definidos de afetividade perante o meio social. A psicomotricidade na parte integral vem desenvolvendo o estímulo corporal durante toda a sessão e faz com quer receba os estímulos necessários de movimentos. Já no artigo de Falkenbach AP et al. (2010), relatam na sua discussão destacando o papel do fisioterapeuta com a criança com o diagnóstico de transtorno do espectro autista na prática psicomotora, onde também é importante destacar que as limitações biológicas do diagnóstico de Autismo não impediram o menino de avançar em sua relação social e em sua trajetória do brincar nas sessões de psicomotricidade. 


\section{CONCLUSÃO}

Portanto pode-se verificar que a Psicomotricidade em crianças com TEA possui benefícios positivos na melhora da qualidade de vida da criança, onde foi possível compreender que o transtorno do espectro autista pode afetar diversos aspectos, tais como, comunicação, interação social, comportamento, desenvolvimento motor, dentre outros. E com o trabalho da Psicomotricidade, podemos observar a melhora no padrão motor e cognitivo da criança melhorando o equilíbrio, marcha, coordenação, interação social e podendo ainda, expressar-se e ser compreendida. Nesse sentido, verificou-se que a Psicomotricidade trabalhará com o corpo em movimento e, paralelamente, com as questões afetivas, tônico-emocionais, cognitivas, sociais e melhora na qualidade de vida.

\section{REFERÊNCIAS}

1. AMERICAN PSYCHIATRIC ASSOCIATION. (2013). Diagnostic and statistical manual of mental disorders (DSM-5®). American Psychiatric Pub.

2. AMERICAN PSYCHIATRIC ASSOCIATION. (2014). DSM-5: Manual diagnóstico e estatístico de transtornos mentais. Artmed Editora.

3. ANDRADE FFD. (2014). Psicomotricidade no ensino infantil: como utilizar o brincar como ferramenta didática?

4. AZEVEDO A, GUSMÃO M. (2016). A importância da fisioterapia motora no acompanhamento de crianças autistas. Rev. Eletrôn. Atualiza Saúde, 2(2), 76-83.

5. BACKES B, et al. (2013). A relação entre regressão da linguagem e desenvolvimento sociocomunicativo de crianças com transtorno do espectro do autismo. In CoDAS (Vol. 25, No. 3, pp. 268-273).

6. CORDEIRO LC, DA SILVA D. (2018). A contribuição da psicomotricidade relacional no desenvolvimento das crianças com transtorno do espectro autista. Faculdade Sant'Ana em Revista, 2(1).

7. CRUZ BDQ, POTTKER C A (2017). As contribuições da equoterapia para o desenvolvimento psicomotor da criança com transtorno de espectro autista. Revista UNINGÁ Review, 32(1), 147-158.

8. DIAS S. (2015). Asperger y su síndrome en 1944 y en la actualidad. Revista Latinoamericana de Psicopatologia Fundamental, 18(2), 307-313.

9. DOS ANJOS CC, et al. (2018). Percepção dos Cuidadores de Crianças com Transtorno do Espectro Autista sobre a Atuação da Fisioterapia. Revista Portal: Saúde e Sociedade, 2(3), 517-532.

10. DOS ANJOS C, et al (2017). Perfil Psicomotor de Crianças com Transtorno do Espectro Autista em Maceió/AL. Revista Portal: Saúde e Sociedade, 2(2), 395-410.

11. DOS ANJOS CC, et al. (2017). Perfil Psicomotor de Crianças com Transtorno do Espectro Autista em Maceió/AL. Revista Portal: Saúde e Sociedade, 2(2), 395-410.

12. DOS SANTOS ÉCF, MÉLO TR. (2018). Caracterização psicomotora de criança autista pela escala de desenvolvimento motor. Divers@!, 11(1), 50-58.

13. FALKENBACH AP, et al (2010). O jogo da criança autista nas sessões de psicomotricidade relacional. Revista Brasileira de Ciências do Esporte, 31(2), 203-214.

14. GONÇALVES IAM. (2012). A Psicomotricidade e as perturbações do espectro do autismo no Centro de Recursos para a Inclusão da APPDA-Lisboa (Doctoral dissertation).

15. KUMAMOTO L. (2012). Autismo-Uma abordagem psicomotora. Psicologia: Teoria e Pesquisa, 5(2), $231-238$.

16. LOCATELLI PB, SANTOS MFR. (2016). Autismo: propostas de intervenção. Revista Transformar, 8(8), $203-220$.

17. RUTTER ML. (2011). Progress in understanding autism: 2007-2010. Journal of autism and developmental disorders, 41(4), 395-404.

18. SANTOS THF, FERNANDES FDM. (2012). Functional Communication Profile-Revised: uma proposta de caracterização objetiva de crianças e adolescentes do espectro do autismo. Revista da Sociedade Brasileira de Fonoaudiologia.

19. SOUZA DC, et al. (2017). A Psicomotricidade aquática com crianças autistas. Encontro Internacional de Formação de Professores e Fórum Permanente de Inovação Educacional, 10(1).

20. TELES PS, CRUZ CLP. (2018). A prática esportiva como instrumento de inclusão: Um estudo de caso sobre aprendizagem e desenvolvimento de aluno com transtorno do espectro autista (TEA). Encontro Internacional de Formação de Professores e Fórum Permanente de Inovação Educacional, 11(1).

21. TRINDADE NG, et al. (2016). A música como auxílio no tratamento fisioterapêutico em pacientes com autismo: estudo de caso. Revista FisiSenectus, 3(2), 3-11.

22. ZIMMER PN, et al. (2018). Educação musical e transtorno do espectro autista: Análise da produção em revistas brasileiras de artes/música qualis a1 e seus anais de eventos regionais e nacionais (2006-2016). Revista da Abem, 26(40). 